\title{
Regulatory roles of miRNA-758 and matrix extracellular phosphoglycoprotein in cervical cancer
}

\author{
XIANHUA MENG ${ }^{1}$, YINGHUI ZHAO ${ }^{1}$, JINYUN WANG ${ }^{1}$, ZHENG GAO $^{1}$, QINGXIA GENG $^{2}$ and XIAOXIA LIU ${ }^{1}$ \\ ${ }^{1}$ Department of Gynaecology and Obstetrics, Laiwu City People's Hospital, Laiwu, Shandong 271199; \\ ${ }^{2}$ Department of Gynaecology and Obstetrics, Laiwu Maternal and Child Care Hospital, Laiwu, Shandong 271100, P.R. China
}

Received March 23, 2016; Accepted March 3, 2017

DOI: $10.3892 /$ etm.2017.4887

\begin{abstract}
The present study aimed to examine the role and underlying mechanism of miRNA-758 (miR-758) expression in cancer tissues, blood and cervical exfoliated cells from patients with cervical cancer. A total of 49 patients with cervical cancer and 26 healthy people for cervical cancer screening were included in the present study. The patients with cervical cancer were treated with resection, and the tumor and adjacent tissues, blood and cervical exfoliated cells were collected. The expression levels of miR-758 and matrix extracellular phosphoglycoprotein (MEPE) mRNA in each sample were detected by reverse transcription-quantitative polymerase chain reaction. In addition, western blot analysis was used to detect the MEPE protein in tumor tissues, while ELISA was applied to detect the MEPE protein expression in the blood and cervical exfoliated cells. Compared with the normal control, MEPE mRNA expression was upregulated in cervical cancer tissues, blood and cervical exfoliated cells. At the protein level, MEPE was also upregulated significantly in patients with cervical cancer. miR-758 expression was decreased significantly in cervical cancer tissues, blood and cervical exfoliated cells $(\mathrm{P}<0.05)$, which was opposite to the trend observed for MEPE mRNA expression. Furthermore, MEPE expression was increased in the tumor tissue, blood and cervical exfoliated cells of cervical cancer patients, which was associated to the downregulated miR-758. Therefore, miR-758 may regulate the infiltration and invasion of cervical cancer by targeting MEPE.
\end{abstract}

\section{Introduction}

Cervical cancer is one of the most common gynecological malignancies, accounting for $10 \%$ of all gynecological

Correspondence to: Dr Qingxia Geng, Department of Gynaecology and Obstetrics, Laiwu Maternal and Child Care Hospital, 12 Fengcheng West Street, Laiwu, Shandong 271100, P.R. China E-mail: cqww33@163.com

Key words: miRNA-758, cervical cancer, matrix extracellular phosphoglycoprotein cancer cases (1). The incidence rate of cervical cancer is $\sim 5 \%$ among all tumors, which follows after the incidence of breast cancer $(2,3)$. There are nearly 500,000 new cases of cervical cancer worldwide each year, and about 270,000 cases of cervical cancer patients succumb to the disease (4). The majority of these mortalities are reported in developing countries (5). In China, the morbidity is growing at 2-3\% each year, and the new cases are approximately one-third of the total incidence in the world. Furthermore, the age of incidence of cervical cancer is gradually decreasing (6).

Currently, the popular treatment for cervical cancer remains surgery and radiotherapy; however, a good response to surgery is more likely among patients at an early stage of the disease (7). Although radiotherapy is suitable for treatment of patients at all stages, it can irreversibly injure the ovaries and vagina, affecting the life quality of young patients (8). Squamous cell carcinoma is the main pathological type of cervical cancer, which accounts for $80-85 \%$ of cases (9). Metastasis typically occurs through direct spreading or lymph node metastasis, and lymph node metastasis is the main mode for distant metastasis (9). Prognosis is also dependent on tumor size, whether the tumor involves the cervical area, and lymph node metastasis (10). Currently, there are no valuable molecular prognosis markers; therefore, it is important to identify prognosis markers and novel targets for cervical cancer therapy.

Matrix extracellular phosphoglycoprotein (MEPE) was identified and cloned from a cDNA library of tumor tissues obtained from patients with oncogenic hypophosphatemic osteomalacia (OHO) by Rowe et al in 2000 (11). Reverse transcription-polymerase chain reaction (RT-PCR) results revealed that MEPE was expressed in the bone mallow and brain, while its expression was lower in the lung, kidney and placenta. MEPE was regarded as a phosphorus regulator inducing $\mathrm{OHO}$, as it was highly expressed in $\mathrm{OHO}$ tumor tissues (12). It was also observed that MEPE served important roles against the resistance of DNA damage and DNA repair processes (13). Furthermore, the expression level of MEPE/OF45 was closely associated with tumor metastasis, which served important roles in drug resistance (14). To the best of our knowledge, no previous studies have investigated MEPE in cervical cancer. In the present study, the expression of MEPE at the mRNA and protein levels was detected in cervical tumor tissues, blood and cervical exfoliated cells by RT-quantitative PCR (RT-qPCR), western blot analysis and ELISA. 
miRNAs play an important role in tumorigenesis and progression by its cleavage function, up-regulating or down-regulating mRNA expression to mediate gene activity of the regulatory proteins $(15,16)$. In the present study, through bioinformatics prediction, it was predicted and validated that MEPE was associated with miRNA-758 (miR-758) expression, and the mechanism underlying the participation of miR-758 in cervical cancer through regulating MEPE was discussed.

\section{Materials and methods}

Clinical data and sample collection. The present study included 49 cervical cancer patients who received surgical resection at the Department of Gynaecology and Obstetrics in Laiwu City People's Hospital (Laiwu, China) between November 2012 and January 2015. In addition, 26 non-tumor patients who underwent cervical cancer screening during the same time period at the same hospital were collected as control group. Biopsies were performed to obtain tumor and adjacent tissues (or non-tumor tissue from control patients). The age of cervical cancer patients ranged between 27 and 69 years, with median age of 48.6 years. Similarly, the age of the control group ranged between 25 and 66 years, with a median age of 45.5 years. All cancer patients included in the present study were treated for the first onset of cancer, and had no history of receiving hormone medication, radiotherapy or chemotherapy prior to surgery. The diagnosis of all patients (17) was confirmed by pathologists in the Laiwu City People's Hospital. In addition, the cervical squamous cell carcinoma pathological grading system (17) was used to classify the differentiation degree of tumors, while metastasis, tumor diameter and depth of invasion were also recorded.

The resected tumor tissues and adjacent normal tissues were preserved in liquid nitrogen. Fasting peripheral blood from the patients in the morning before surgery was collected, placed in EDTA anticoagulant and stored at $-20^{\circ} \mathrm{C}$. Cervical exfoliated cells were also collected with a special soft brush and placed in a preservation solution. Prior written informed consent was obtained from each patient, and the study was approved by the Ethics Review Board of the Laiwu City People's Hospital.

MEPE $m R N A$ expression detection by RT-qPCR. Total RNA was isolated from the resected tissues, peripheral blood and cervical exfoliated cells using $1 \mathrm{ml}$ TRIzol reagent (Invitrogen; Thermo Fisher Scientific, Inc., Waltham, MA, USA) per $100 \mathrm{mg}$ of tissue, according to the manufacturer's protocol. The phenol-chloroform method was used to extract the total RNA. Next, the RNA integrity was checked by $1 \%$ agarose gel electrophoresis, and the purity of RNA was determined by spectrophotometric detection of absorbance at 260/280 nm. Under the UV light, three bands of 28,18 , and $5 \mathrm{sec}$ were observed, and the ratio of 28/18 sec was 2:1. cDNA was then synthesized from total RNA by the Reverse TIANScript II cDNA kit (cat. no. KR107; Tiangen Biotech Co., Ltd., Beijing, China) and stored in $-20^{\circ} \mathrm{C}$. The primers used in qPCR were as follows: MEPE forward, 5'-ATGCACACTCCCGACAGA GGA-3' and reverse, 5'-CCTTGATGAGCATTTTGGCTG CT-3'; GAPDH forward, 5'-TGACCTTGCCCACAGCCT TG-3', and reverse, 5'-CATCACCATCTTCCAGGAGCG-3'. The SYBR-Green qPCR analysis was performed using the SuperReal PreMix (Tiangen Biotech Co., Ltd.). The cycle conditions were the following: $95^{\circ} \mathrm{C}$ for $5 \mathrm{~min}$, followed by 35 cycles at $95^{\circ} \mathrm{C}$ for $30 \mathrm{sec}, 55^{\circ} \mathrm{C}$ for $30 \mathrm{sec}$ and $72^{\circ} \mathrm{C}$ for $60 \mathrm{sec}$. The relative expression of MEPE was calculated using the $2^{-\Delta \Delta C q}$ method (18), compared with GAPDH as an internal control.

Western blot analysis. Total proteins were extracted from tissues and cells based on the standard protocol of protein lysis (cat. no. R6731-00; Omega Bio-Tek, Inc., Norcross, GA, USA). The protein concentration was detected using a BCA protein concentration assay kit (cat. no. P0009; Beyotime Institute of Biotechnology, Haimen, China). In total, $20 \mu \mathrm{g}$ protein was mixed with equal volume of $2 X$ SDS loading buffer and boiled for $5 \mathrm{~min}$. Next, the protein was subjected to $10 \%$ SDS-PAGE $(100 \mathrm{~V}$ constant voltage for $2 \mathrm{~h}$ ) and then transferred to a polyvinylidene fluoride membrane (in ice incubation). Subsequent to blocking with skim milk, the protein sample was incubated with primary rabbit anti-human MEPE antibody (1:1,000; ab108073; Abcam, Cambridge, MA, USA) and rabbit anti-human $\beta$-actin antibody $\left(1: 5,000\right.$; ab6276; Abcam) at $4^{\circ} \mathrm{C}$ overnight. The samples were then incubated with a horseradish peroxidase-conjugated goat anti-rabbit IgG secondary antibody (1:3,000; ab6721; Abcam) at room temperature for $1 \mathrm{~h}$. All the antibodies were purchased from Abcam (Cambridge, MA, USA). Finally, the membrane was developed using an Enhanced Chemiluminescence Plus reagent (cat. no. P0018; Beyotime Institute of Biotechnology). The developed film was scanned and analyzed by Image Lab version 3.0 software (Bio-Rad Laboratories, Inc., Hercules, CA, USA). The grey intensity of $\beta$-actin was used as an internal control to calculate the relative expression of MEPE protein.

ELISA. The peripheral blood was centrifuged at $1,200 \mathrm{x} g$ for 10 min to separate the serum and red blood cells. In addition, total proteins were extracted from the cervical exfoliated cells with a protein lysis solution based on the standard protocol, and the BCA method was used to estimate the protein concentration. Subsequently, the serum and the protein lysate of cervical exfoliated cells were used to determine the MEPE protein expression according to the manufacturer's instructions of the ELISA kit (cat no. 0227; Baili Biological Technology Co., Ltd., Shanghai, China). Briefly, $50 \mu 1$ of standards (with different standard concentrations as instructed by the kit) was added into wells on the ELISA plate, and $10 \mu \mathrm{l}$ of sample was diluted to $50 \mu \mathrm{l}$. A standard curve was generated. With the exception of blank wells, $100 \mu \mathrm{l}$ HRP-labeled antibody was added to each well, covered with microplate sealers and incubated for $1 \mathrm{~h}$. After washing with PBS five times, $50 \mu 1$ substrate A and $50 \mu 1$ substrate B were added into each well. Following incubation at $37^{\circ} \mathrm{C}$ for $15 \mathrm{~min}$, $50 \mu 1$ stop solution was added into each well. The optical density value was measured at a wavelength of $450 \mathrm{~nm}$ within $15 \mathrm{~min}$.

Bioinformatics prediction. Bioinformatics prediction is the basis for studying the function of miRNAs in various diseases. In the present study, bioinformatics algorithms were applied to predict candidate miRNAs that are able of direct complementary binding to the 3'-untranslated region of MEPE. A total of 10 software tools were used, five of which indicated a positive prediction for miR-758. That is, miRanda (www.microrna. org/microrna/getExprForm.do), TargetScan (www.targetscan. 
A

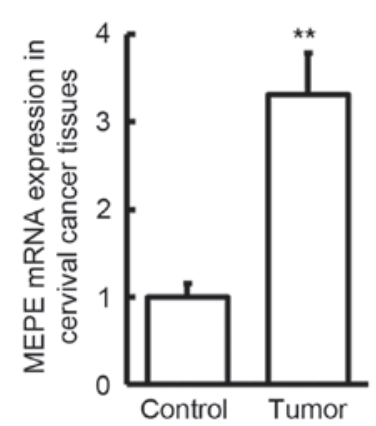

B

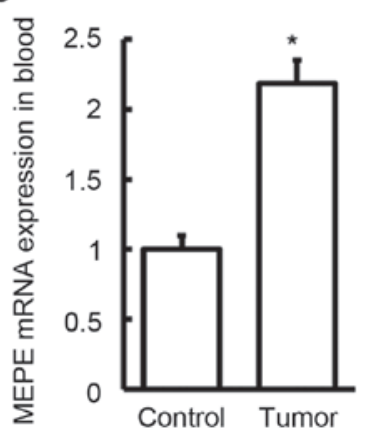

C

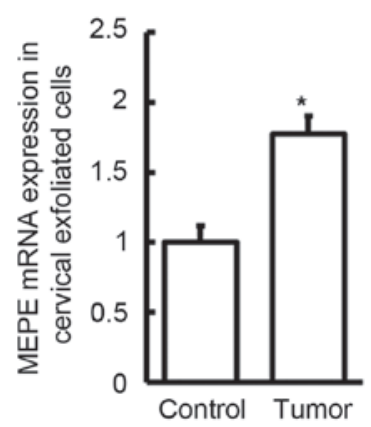

Figure 1. Expression of MEPE mRNA in (A) cervical tissue samples, (B) blood samples and (C) cervical exfoliated samples from healthy controls and cervical cancer patients. The expression of MEPE was detected using reverse transcription-quantitative polymerase chain reaction. MEPE expression was increased in the tumor tissues, blood and cervical exfoliated cells of cancer patients compared with the normal control group. ${ }^{*} \mathrm{P}<0.05$ and ${ }^{* *} \mathrm{P}<0.01$, vs. control group. MEPE, matrix extracellular phosphoglycoprotein.

org), PITA (genie.weizmann.ac.il/pubs/mir07/mir07_data. html), RNAhybrid (bibiserv.techfak.uni-bielefeld.de/rnahybrid) and PiCTA (pictar.mdc-berlin.de) software tools identified that miR-758 was one of the miRNAs that directly regulates MEPE. The five additional tools that indicated no positive prediction for miR-758 were DIANA-mT (diana.imis.athena-innovation. gr/DianaTools/index.php), miRWalk (zmf.umm.uni-heidelberg. de/apps/zmf/mirwalk/index.html), RNA22 (cm.jefferson. edu/rna22/Interactive), miRDB (www.mirdb.org) and miRbase (www.mirbase.org).

miRNA RT-qPCR. Total miRNAs were isolated from tumor samples, peripheral blood and cervical exfoliated cells based on the manufacturer's protocol of miRcute miRNA Isolation kit (Tiangen Biotech Co., Ltd.). cDNA was reverse transcribed using the miRcute miRNA cDNA kit (Tiangen Biotech Co., Ltd.), and miR-758 was then detected by the miRcute miRNA luciferase quantitative kit (Tiangen Biotech Co., Ltd.). U6 was used as an internal reference. The following primers were used: miR-758 forward, 5'-ACACTCCAGCTGGGAACGATG-3', and reverse, 5'-CTCAACTGGTGTCGTGGAGTCGGCA-3'; U6 forward, 5'-CTCGCTTCGGCAGCACA-3', and reverse, 5'-AACGCTTCACGAATTTGCGT-3'. The thermal cycling conditions were the following: $95^{\circ} \mathrm{C}$ for $5 \mathrm{~min}$, followed by 40 cycles at $95^{\circ} \mathrm{C}$ for $15 \mathrm{sec}, 60^{\circ} \mathrm{C}$ for $30 \mathrm{sec}$, and $72^{\circ} \mathrm{C}$ for $20 \mathrm{sec}$. The relative expression was calculated with the $2^{-\Delta \Delta \mathrm{Cq}}$ method, on the basis of the grey intensity ratio of miR-758/U6.

Statistical analysis. All the data are presented as the mean \pm standard deviation, and were analyzed by SPSS version 18.0 software (SPSS, Inc., Chicago, IL, USA). For comparisons among multiple groups, one-way analysis of variance was applied. The least significant difference and Student-Newman-Keuls methods were used in cases of homogeneity of variance, while Tamhane's T2 or Dunnett's T3 method was applied when heteroscedasticity was observed. Differences with $\mathrm{P}<0.05$ were considered to be statistically significant, with $\mathrm{P}<0.01$ regarded as strong significance.

\section{Results}

Patient characteristics. Among the 49 cancer patients, 35 highly-differentiated and 14 lowly-differentiated cases were
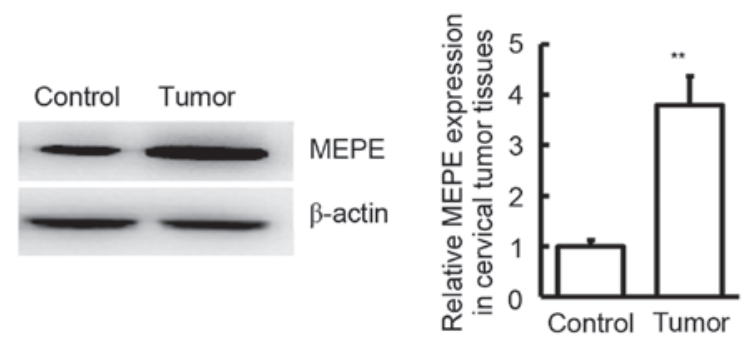

Figure 2. Western blot analysis was performed to detect MEPE expression in cervical cancer tissues. MEPE was increased in cervical tumor tissues compared with the normal tissues. ${ }^{* *} \mathrm{P}<0.01$ vs. control group. MEPE, matrix extracellular phosphoglycoprotein.

included, as classified with the cervical squamous cell carcinoma pathological grading system. Furthermore, 16 cases had lymph node metastasis, while the remaining 33 cases did not present metastasis. Regarding the tumor size, the tumor diameter in 8 cases was $>4 \mathrm{~cm}$, while the diameter in the remaining 41 patients were $<4 \mathrm{~cm}$. Regarding the depth of invasion, the tumor invaded the superficial muscle in 26 cases and the deep myometrial in 23 cases.

Expression of MEPE $m R N A$ in different cervical tumor samples. To detect the alteration of MEPE mRNA expression in different samples, RT-qPCR was applied. As shown in Fig. 1, MEPE mRNA was significantly upregulated in cervical tumor tissues (Fig. 1A), blood (Fig. 1B) and cervical exfoliated cells (Fig. 1C), when compared with the adjacent normal tissues, normal blood and cervical exfoliated cells from non-tumor patients, respectively $(\mathrm{P}<0.05)$. The results indicated that MEPE may serve a regulatory role in cervical cancer.

Expression of MEPE protein in tumor samples. To examine the expression of MEPE protein in cervical cancer tumor tissues, western blot analysis was used in the present study. Compared with the adjacent control, MEPE protein expression was significantly upregulated in cervical cancer tissues $(\mathrm{P}<0.05)$, as shown in Fig. 2 . The results demonstrated that the increase of MEPE protein was consistent with the trend observed for the mRNA level, which indicated that overexpression of MEPE served a regulatory role in cervical cancer. 

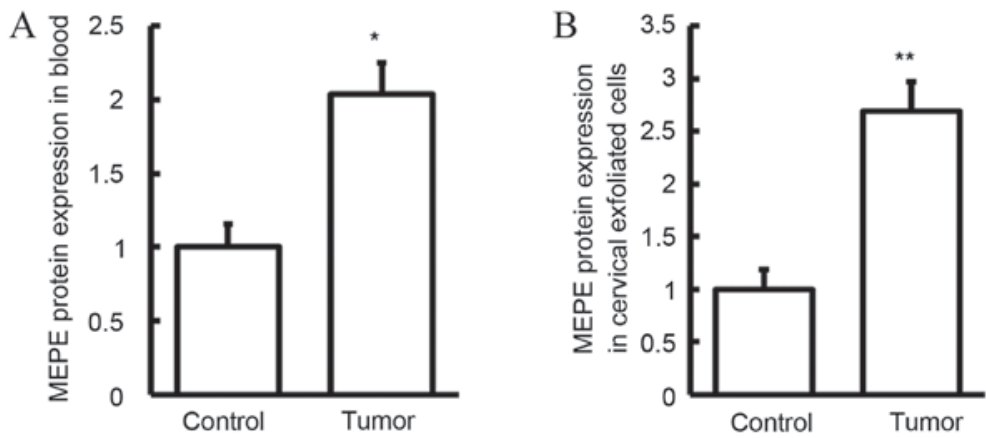

Figure 3. ELISA method was performed to detect the MEPE protein expression in the (A) blood and (B) cervical exfoliated cells of cervical tumor patients and healthy controls. MEPE protein was increased in the blood and cervical exfoliated cells of cervical tumor patients compared with the control. "P<0.05 and ${ }^{* *} \mathrm{P}<0.01$, vs. control group. MEPE, matrix extracellular phosphoglycoprotein.

A

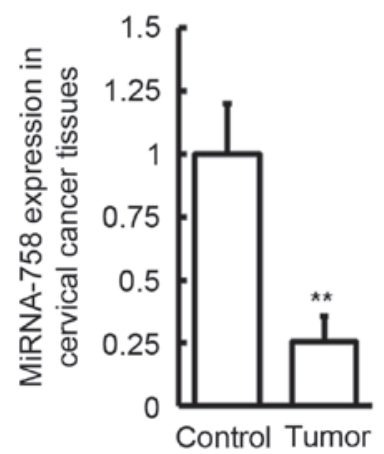

B

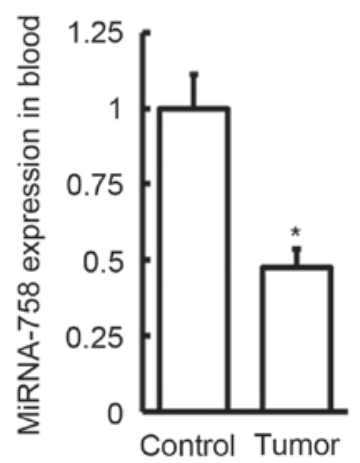

C

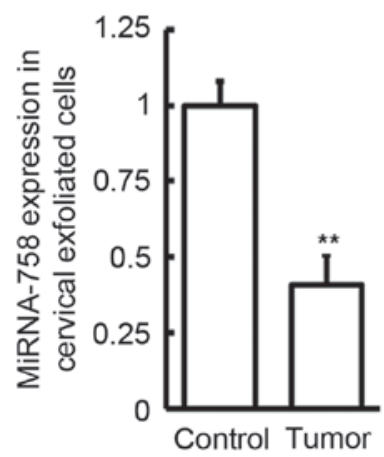

Figure 4. Expression of miRNA-758 in (A) cervical tissue samples, (B) blood samples and (C) cervical exfoliated samples from healthy controls and cervical cancer patients. The expression of miRNA-758 was detected using reverse transcription-quantitative polymerase chain reaction. miRNA-758 expression was decreased in the three types of samples from cervical cancer patients when compared with normal control samples. ${ }^{*} \mathrm{P}<0.05$ and ${ }^{* *} \mathrm{P}<0.01$, vs. control group. MEPE, matrix extracellular phosphoglycoprotein.

Expression of MEPE protein in the blood and cervical exfoliated cells of cervical cancer patients. To examine MEPE protein expression in the peripheral blood and cervical exfoliated cells of cervical cancer patients, ELISA was performed. Compared with the associated controls, MEPE protein expression in the blood and cervical exfoliated cells of cervical cancer patients was significantly increased $(\mathrm{P}<0.05)$, as shown in Fig. 3. The results demonstrated that the increase in MEPE protein was consistent with that in the mRNA level in the tumor samples, blood and cervical exfoliated cells. Since blood is a common channel for tumor metastasis, increased MEPE protein in the blood indicated that MEPE may influence the metastasis of cervical cancer. Upregulation of MEPE in cervical exfoliated cells might be a potential biomarker for cervical cancer.

miR-758 expression in different samples. To detect the miR-758 expression among different samples, RT-qPCR was used to analyze tumor tissues, blood samples and cervical exfoliated cells obtained from cervical cancer patients and healthy controls. Compared with the control group, miR-758 was significantly downregulated in cervical cancer patients $(\mathrm{P}<0.05)$, as shown in Fig. 4. Combined with the bioinformatics prediction that miR-758 directly regulates MEPE, it can be inferred that miR-758 served an important role in the pathological processes of cervical cancer. The results indicated that miR-758 exerted its function through regulating MEPE mRNA and consequently inducing MEPE protein expression.

\section{Discussion}

In the present study, MEPE expression at the mRNA and protein levels was investigated in tumor tissues, blood samples and cervical exfoliated cells of cervical cancer patients, compared with healthy control samples. The expression of miR-758 was also explored in these specimens, and the mechanisms underlying the effect of miR-758 expression in cervical cancer through regulation of MEPE were discussed.

Cervical cancer is the second most common malignancy in women after breast cancer world widely (19). Cervical cancer screening helps detection at an early stage and improves the prognosis and treatment of cervical cancer. However, invasion and metastasis pose a challenge in the treatment of cervical cancer. Lymph node metastasis is the most common type of remote metastasis and is closely associated with poor prognosis. Identifying this metastasis can help to judge prognosis and treatment strategies (20). Deep understanding of lymph node metastasis and lymphangiogenesis in cervical cancer is helpful to identify novel and effective treatment methods.

Currently, cervical cytological examination alone or in combination with human papilloma virus (HPV) detection are usually used for cervical cancer screening (21). A previous 
study indicated that cervical cytological examination was effective to classify HPV-positive women (22). The main advantage of cervical cytology is high specificity, so the EUROGIN organization recommended the use of cervical cytological examination for stratifying HPV infected women for preliminary screen (23). In the present study, one of the samples collected was cervical exfoliated cells, and molecular detection on these cells provided the basis for diagnosis and treatment of cervical cancer.

MEPE/OF45 is thought to be associated with bone metabolism. Several studies indicated that MEPE/OF45 served important roles in bone regeneration, differentiation and maturation (24-26). In addition, it has been demonstrated that MEPE was highly expressed in tumor-induced osteomalacia (27) and SaOS2 osteosarcoma cells (28). Due to the unique structural character and role in tumor at different stages, MEPE has become an important molecular tool for studying tumor development and angiogenesis (15). MEPE serves roles against DNA damage and can repair DNA damage, which directly causes drug resistance in tumors (13). It has been also observed that MEPE expression was highly correlated with the metastasis of tumor cells (14). In the present study, MEPE was significantly downregulated in tumor tissues, which was consistent with the authors' predictions. The results demonstrated that MEPE expression was increased not only in tumor tissues, but also in the blood of cervical cancer patients. Besides local infiltration, tumor cells can be spread through the blood, thus MEPE expression alteration in the blood also indicated that it may be involved in tumor metastasis. In the present study, the MEPE expression in cervical exfoliated cells that are typically used in cervical cytological examination was also detected. It was found that MEPE expression was also increased in cervical exfoliated cells, which indicated that MEPE may be a potential biomarker for cervical cancer, particularly at the early stage of the disease.

To further investigate the regulation mechanisms of MEPE, bioinformatics tools were applied to predict upstream regulators of MEPE. It was previously reported that endogenous miRNAs can degrade MEPE mRNA and inhibit its translation (16). Through degrading mRNA of target genes, miRNAs serve important roles in tumor development and progression $(29,30)$. miR-376a has been reported to regulate MEPE, which increased the sensitivity of tumor cells to chemotherapy and radiotherapy (31). In the present study, through bioinformatics prediction, miR-758 expression was demonstrated to be closely associated with MEPE, and thus miR-758 may be another miRNA that regulates MEPE. miR-758 has been reported to be abnormally expressed in various diseases. For instance, during chemotherapy in esophageal cancer, miRNA-758 expression was repressed by chemotherapy drugs, which indicated that the dysfunction of downstream target genes was associated with the drug resistance (32). miR-758 also participated in the atherosclerotic process by regulating the expression of toll-like receptors (33). In addition, miR-758 regulated cholesterol efflux through inhibiting ABCA1 (34). In the present study, miR-758 was downregulated in cervical tumor tissues, blood samples and cervical exfoliated cells, which may indicate that increased MEPE was regulated by miR-758. The balance of upregulated expression of MEPE and decreased miR-758 participated in the development and progression of cervical cancer. Furthermore,
miR-758 may be more suitable as a diagnostic biomarker as it is stably expressed in blood and cervical exfoliated cells.

The occurrence and development of cervical cancer is a complex process, which involves multiple factors, mutation accumulation of multiple genes and various molecular interactions. The expression changes of various oncogenes, tumor suppressor genes and metastasis-associated genes interact together to induce cervical cancer (35). The present study results of miR-758 and MEPE expression levels in the tumor, blood and cervical exfoliated cells indicated that their levels are sensitive and specific to cervical cancer. As blood and cervical exfoliated cells can be easily obtained without invasion, detection of miRNA-75 and MEPE in these cells may be used as supplementary diagnostic biomarkers in cervical cancer screening. However, it is still necessary to further explore the biological function of miR-758 in cervical cells by obtaining direct evidence of how miR-758 regulates MEPE in cervical cancer.

In conclusion, the balancing between miR-758 and MEPE plays a role in the development and progression of cancer, and miR-758 may serve as a biomarker for early diagnosis of cervical cancer. The results also provide a new hypothesis that miR-758 negatively regulates MEPE, which may aid the diagnosis, prevention and treatment of cervical cancer.

\section{Acknowledgements}

The authors would like to thank Professor Xiaohong Wang from the Department of Gynaecology and Obstetrics, Laiwu City People's Hospital (Laiwu, China) and Professor Yu Deng from the Department of Pathology, Laiwu City People's Hospital, for their suggestions on the experimental design, results analysis and manuscript revision.

\section{References}

1. Hovland S, Muller S, Skomedal H, Mints M, Bergström J, Wallin KL, Karlsen F, Johansson B and Andersson S: E6/E7 mRNA expression analysis: A test for the objective assessment of cervical adenocarcinoma in clinical prognostic procedure. Int J Oncol 36: 1533-1539, 2010.

2. de Martel C, Ferlay J, Franceschi S, Vignat J, Bray F, Forman D and Plummer M: Global burden of cancers attributable to infections in 2008: A review and synthetic analysis. Lancet Oncol 13: 607-615, 2012.

3. Ferlay J, Shin HR, Bray F, Forman D, Mathers C and Parkin DM: Estimates of worldwide burden of cancer in 2008: GLOBOCAN 2008. Int J Cancer 127: 2893-2917, 2010.

4. Wolfson IN: Letter: Blind defibrillation. Am J Cardiol 36: 412, 1975.

5. Sankaranarayanan R and Ferlay J: Worldwide burden of gynaecological cancer: The size of the problem. Best Pract Res Clin Obstet Gynaecol 20: 207-225, 2006.

6. Arbyn M, Castellsagué X, de Sanjosé S, Bruni L, Saraiya M, Bray F and Ferlay J: Worldwide burden of cervical cancer in 2008. Ann Oncol 22: 2675-2686, 2011.

7. Andrae B, Andersson TM, Lambert PC, Kemetli L, Silfverdal L, Strander B, Ryd W, Dillner J, Törnberg S and Sparén P: Screening and cervical cancer cure: Population based cohort study. BMJ 344: e900, 2012.

8. Park SY, Bae DS, Nam JH, Park CT, Cho CH, Lee JM, Lee MK, Kim SH, Park SM and Yun YH: Quality of life and sexual problems in disease-free survivors of cervical cancer compared with the general population. Cancer 110: 2716-2725, 2007.

9. Voulgari A and Pintzas A: Epithelial-mesenchymal transition in cancer metastasis: Mechanisms, markers and strategies to overcome drug resistance in the clinic. Biochim Biophys Acta 1796: 75-90, 2009. 
10. Lee DW, Kim YT, Kim JH, Kim S, Kim SW, Nam EJ and Kim JW: Clinical significance of tumor volume and lymph node involvement assessed by MRI in stage IIB cervical cancer patients treated with concurrent chemoradiation therapy. J Gynecol Oncol 21: 18-23, 2010.

11. Rowe PS, de Zoysa PA, Dong R, Wang HR, White KE, Econs MJ and Oudet CL: MEPE, a new gene expressed in bone marrow and tumors causing osteomalacia. Genomics 67: 54-68, 2000.

12. Bresler D, Bruder J, Mohnike K, Fraser WD and Rowe PS: Serum MEPE-ASARM-peptides are elevated in X-linked rickets (HYP) Implications for phosphaturia and rickets. J Endocrinol 183 : R1-R9, 2004.

13. Sheng J, Luo W, Yu F, Gao N and Hu B: MicroRNA-376a sensitizes cells following DNA damage by downregulating MEPE expression. Cancer Biother Radiopharm 28: 523-529, 2013.

14. Zhang P, Wang H, Rowe PS, Hu B and Wang Y: MEPE/OF45 as a new target for sensitizing human tumour cells to DNA damage inducers. Br J Cancer 102: 862-866, 2010.

15. Lamour V, Nokin MJ, Henry A, Castronovo V and Bellahcène A: SIBLING proteins: Molecular tools for tumor progression and angiogenesis. Med Sci (Paris) 29: 1018-1025, 2013 (In French).

16. Liu K, Liu S, Zhang W, Jia B, Tan L, Jin Z and Liu Y: miR-494 promotes cell proliferation, migration and invasion, and increased sorafenib resistance in hepatocellular carcinoma by targeting PTEN. Oncol Rep 34: 1003-1010, 2015.

17. Hillemanns P, Soergel P, Hertel H and Jentschke M: Epidemiology and early detection of cervical cancer. Oncol Res Treat 39: 501-506, 2016.

18. Livak KJ and Schmittgen TD: Analysis of relative gene expression data using real-time quantitative PCR and the 2(-Delta Delta C(T)) method. Methods 25: 402-408, 2001.

19. Hakama M, Coleman MP, Alexe DM and Auvinen A: Cancer screening: Evidence and practice in Europe 2008. Eur J Cancer 44: 1404-1413, 2008.

20. Heeren AM, de Boer E, Bleeker MC, Musters RJ, Buist MR, Kenter GG, de Gruijl TD and Jordanova ES: Nodal metastasis in cervical cancer occurs in clearly delineated fields of immune suppression in the pelvic lymph catchment area. Oncotarget 6 : 32484-32493, 2015

21. Kotaniemi-Talonen L, Nieminen P, Anttila A and Hakama M: Routine cervical screening with primary HPV testing and cytology triage protocol in a randomised setting. Br J Cancer 93 862-867, 2005

22. Franceschi S, Denny L, Irwin KL, Jeronimo J, Lopalco PL, Monsonego J, Peto J, Ronco G, Sasieni P and Wheeler CM: Eurogin 2010 roadmap on cervical cancer prevention. Int J Cancer 128: 2765-2774, 2011

23. Harris SE, Gluhak-Heinrich J, Harris MA, Yang W, Bonewald LF, Riha D, Rowe PS, Robling AG, Turner CH, Feng JQ, et al: DMP1 and MEPE expression are elevated in osteocytes after mechanical loading in vivo: Theoretical role in controlling mineral quality in the perilacunar matrix. J Musculoskelet Neuronal Interact 7 : $313-315,2007$
24. Siggelkow H, Schmidt E, Hennies B and Hufner M: Evidence of downregulation of matrix extracellular phosphoglycoprotein during terminal differentiation in human osteoblasts. Bone 35 : 570-576, 2004

25. Argiro L, Desbarats M, Glorieux FH and Ecarot B: Mepe, the gene encoding a tumor-secreted protein in oncogenic hypophosphatemic osteomalacia, is expressed in bone. Genomics 74: 342-351, 2001.

26. Lu C, Huang S, Miclau T, Helms JA and Colnot C: Mepe is expressed during skeletal development and regeneration. Histochem Cell Biol 121: 493-499, 2004.

27. Imanishi Y, Hashimoto J, Ando W, Kobayashi K, Ueda T, Nagata Y, Miyauchi A, Koyano HM, Kaji H, Saito T, et al: Matrix extracellular phosphoglycoprotein is expressed in causative tumors of oncogenic osteomalacia. J Bone Miner Metab 30: 93-99, 2012.

28. Prideaux M, Wijenayaka AR, Kumarasinghe DD, Ormsby RT, Evdokiou A, Findlay DM and Atkins GJ: SaOS2 osteosarcoma cells as an in vitro model for studying the transition of human osteoblasts to osteocytes. Calcif Tissue Int 95: 183-193, 2014

29. Lewis BP, Burge CB and Bartel DP: Conserved seed pairing, often flanked by adenosines, indicates that thousands of human genes are microRNA targets. Cell 120: 15-20, 2005.

30. Chen K and Rajewsky N: The evolution of gene regulation by transcription factors and microRNAs. Nat Rev Genet 8: 93-103, 2007.

31. Sheng J, Luo W, Yu F, Gao N and Hu B: MicroRNA-376a sensitizes cells following DNA damage by downregulating MEPE expression. Cancer Biother Radiopharm 28: 523-529, 2013.

32. Hummel R, Wang T, Watson DI, Michael MZ, Van der Hoek M, Haier J and Hussey DJ: Chemotherapy-induced modification of microRNA expression in esophageal cancer. Oncol Rep 26: 1011-1017, 2011.

33. Yang Q, Fu S and Wang J: Hepatitis C virus infection decreases the expression of Toll-like receptors 3 and 7 via upregulation of miR-758. Arch Virol 159: 2997-3003, 2014.

34. Ramirez CM, Davalos A, Goedeke L, Salerno AG, Warrier N, Cirera-Salinas D, Suárez Y and Fernández-Hernando C: MicroRNA-758 regulates cholesterol efflux through posttranscriptional repression of ATP-binding cassette transporter A1. Arterioscler Thromb Vasc Biol 31: 2707-2714, 2011.

35. Zhao S: Specific type epigenetic changes in cervical cancers. Methods Mol Biol 1238: 733-749, 2015. 